\title{
Una revisión histórica del concepto de calor: algunas implicaciones para su aprendizaje
}

\author{
Francisco Javier Camelo Bustos* \\ Sindy Julieth Rodríguez Sotelo** \\ Artículo recibido: 13-4-2007 y aprobado: 30-4-2008
}

A historical revision of the heat concept: some implications for its learning

Resumen: La historia en diversos ámbitos de nuestra cultura, desde el arte, la religión y la política, hasta la técnica y la ciencia, se considerada de vital importancia. En este artículo se intenta aclarar qué aportes puede brindar la historia de la física a la enseñanza y aprendizaje de la misma. Para facilitar este análisis, se expondrá, a manera de ejemplo, la evolución histórica del concepto de calor, haciendo énfasis en cómo utilizar tanto los aciertos como las dificultades que enfrentaron -y enfrentanlos físicos en la elaboración de la idea del calor para dinamizar la enseñanza y aprendizaje de este concepto en contextos escolares.

Palabras clave: Calor, historia de la física, historia del calor, aprendizaje de la física.
Abstract: History is considered of vital importance in different contexts of our culture such as art, religion, politics, technique and science. This article presents the contributions that physics history offers to its teaching and learning processes. To make this analysis easier, it will be taken the historical evolution of the concept of "heat" as an example, emphasizing the proper use of the strengths and drawbacks found by the physicians when facing the task of elaborating the idea of "heat", in order to give dynamics to the teaching and learning of this concept in educational contexts.

Key words: Heat, physics history, heat's history, physics learning.

Profesor de la Secretaría de Educación del Distrito Capital y catedrático de la Universidad Pedagógica Nacional, Colombia.fcamelo1@yahoo.com

** Estudiante, Universidad Distrital Francisco José de Caldas, Colombia. Sindyjuliethr@yahoo.es 


\section{Introducción}

Este documento hace parte de un estudio más amplio, que pretende realizar una mirada histórico epistemológica del calor para diseñar situaciones de aula que posibiliten aprendizajes más significativos en estudiantes de secundaria. Aquí se da cuenta únicamente del desarrollo histórico-epistemológico, dejando para futuras disertaciones el diseño y la aplicación de las situaciones que de tal mirada se deriven. Por tal razón en el primer aparte se presentan consideraciones sobre la importancia de la historia de las ciencias en la enseñanza de las mismas, lo cual genera un contexto para mostrar. En el segundo aparte, la evolución del concepto de calor a través de los tiempos, lo cual posibilita, en las conclusiones, plantear aspectos relevantes a considerar en el diseño de situaciones de aula.

\section{La historia de la física, una consideración imprescindible en la enseñanza de la misma}

Conocer ampliamente la historia de la física posibilita una comprensión más profunda de los conceptos y de los métodos usados, al develar sus orígenes, su evolución y su estado actual; al mismo tiempo, ofrece una visión cultural de los mismos, ya que pone de relieve el rostro y las vidas de quienes fueron sus constructores. Bajo esta mirada, la física aparece como una ciencia viva, ligada a circunstancias históricas y culturales, que no puede prescindir de los problemas de la humanidad. El análisis de la evolución histórica proporciona, además, principios de base sobre cómo ha de enseñarse y aprenderse esta ciencia (Anaconda, 2003 y Cubillos, 2002), los cuales habrá de complementarse desde diferentes disciplinas, como la psicología del aprendizaje, la reflexión sobre la enseñanza, etc. (Del Río, 1997).

Cabe entonces preguntarse, ¿qué aportes puede brindar la historia de la física a la enseñanza de la misma? y ¿cómo puede usarse tal historia para mejorar los ambientes que se dan en el aula de clase?

Para abordar estas preguntas, en primer lugar, se hace necesario aceptar que la historia ha mostrado que las llamadas ciencias exactas, en particular la física, no son solo una construcción lógica y racional, como ha sido popularmente pensada en los últimos tiempos, sino que ella es una construcción sociocultural, producto del trabajo de cientos de mujeres y hombres en contextos históricos determinados (Cubillos, 2002). Esta idea rompe con viejas concepciones que dan a la física tan solo un carácter formal y abstracto que incluso le otorga la incapacidad para cometer errores, implicando que la enseñanza de las mismas se dé de manera rígida y poco atractiva para los estudiantes, pues desconoce diversos aspectos de su construcción.

Por su parte, aceptar una concepción de la física como construcción sociocultural permitirá propender "[...] por una enseñanza dinámica en la que se replantean constantemente tanto los contenidos, como las maneras de comunicarlos [...]" (Anaconda, 2003: 36), lo que a su vez cambia la interacción que se da entre estudiantes y profesor en el salón de clases, posibilitando que el conocimiento se estudie como un objeto en constante construcción, el cual es falible y cuestionable. Álvarez (2005: 2) ilustra claramente este hecho al mencionar que la utilización de la historia posibilita 
[...] cuestionar una enseñanza organizada, casi en exclusiva, alrededor de la presentación de resultados, de conceptos ya construidos. [Pues] debe trabajar sobre el origen de los saberes, ya que estos responden a problemas y preguntas planteadas que se han ido interpretando de manera diferente durante un largo proceso. La ciencia no ha surgido ex nihilo. Ha de abrir una reflexión sobre las disciplinas y los instrumentos intelectuales elaborados para explicar la realidad, como leyes, teorías, modelos o conceptos, mostrando que estos no se han construido de forma acumulativa, sino que son producto de reorganizaciones sucesivas.

Sin embargo, las ideas anteriores son tan solo una mirada de lo que podría implicar una visión sociocultural de la construcción de los fenómenos físicos, pues existen dos posibles escenarios donde aplicar tal reflexión histórica: i) en la formación de profesores y, ii) en el aprendizaje de la física (Anaconda, 2003). En cuanto al aprendizaje de la físi$\mathrm{ca}^{1}$, puede mencionarse que sirve para:

a) Aportar situaciones y problemas interesantes para un tratamiento lúdico, que permita ganar el interés de manera informal sobre un contenido específico. Es decir, aportar problemas que generen en los estudiantes cierta tensión epistémica, un cierto deseo por el saber que ponga en marcha el aprendizaje. Es claro que los problemas llevados al aula no podrán ser literalmente los que originaron el

1 En este artículo solo se desarrolla el segundo escenario, por considerar una tesis con respecto al aprendizaje de la física. El lector interesado en la formación de profesores puede consultar a Quintanilla (2005) y García (2005). concepto o procedimiento, es necesario reformularlos o buscar otros cercanos a lo habitual, a lo ya conocido, pero de cualquier modo problemas desafiantes y novedosos.

b) Servir de puente entre la cultura y la física, pues permite a los individuos comprender de un modo más claro manifestaciones del entorno en el que se encuentran.

A continuación se muestra una reflexión en torno a la evolución histórica del calor, haciendo énfasis en aquellos aspectos relevantes que posibilitarían el diseño de situaciones interesantes, novedosas y con sentido para los estudiantes, ejemplificando, de algún modo, el uso de la historia de la física en el aprendizaje de la misma.

\section{Una reflexión histórica acerca del concepto de calor}

El análisis del concepto de calor permite identificar diversas clasificaciones de su evolución; aquí se consideran cinco momentos históricos relevantes a saber: i) los cuatro elementos, ii) el alcahesto, iii) el flogisto, iv) el calórico, y v) la energía.

\section{Los cuatro elementos}

Este momento comienza por la manifestación más elemental de este fenómeno, el fuego, por el cual el hombre mostró interés desde épocas remotas. Quizá la primera referencia formal para dar cuenta de la importancia del fuego se encuentra en las ideas de Heráclito (540 a.C.-475 a.C.), quien sostenía que el fuego era el origen primordial de la materia y que el mundo entero se encontraba en un estado constante de cambio, postulando al fuego como la sustancia primordial o 
principio que, a través de la condensación y rarefacción ${ }^{2}$ crea los fenómenos del mundo sensible. Como él, muchos filósofos griegos basan sus ideas cosmológicas en el fuego (Taton, 1972).

Este mismo autor señala que Empédocles (493 a.C.-433 a.C.) propone que el mundo se compone de cuatro elementos fundamentales: agua, aire, fuego y tierra, y dos fuerzas opuestas: amor y odio, que actúan sobre estos elementos, combinándolos y separándolos dentro de una variedad infinita de formas. Sosteniendo que no es posible la creación de nueva materia, pues solo pueden ocurrir cambios en las combinaciones de los cuatro elementos primarios.

Estas ideas, aunque tan solo cualitativas, se mantuvieron por más de 23 siglos, siendo completadas por Aristóteles (384 a.C.-322 a.C.), quien agregó dos pares de cualidades fundamentales: caliente y frió, seco y húmedo. La unión de estas dos cualidades correspondía a los cuatro elementos; así, el fuego es cálido y seco, el aire, cálido y húmedo, la tierra, fría y seca, y el agua fría y húmeda. Por tanto, la razón por la cual un cuerpo tenía cierta temperatura la revelaba la cantidad que en él se encontraban estas dos cualidades fundamentales. Galeno (129-199) propuso una escala cualitativa que costaba de cuatro estados de calor y cuatro de frío, el punto neutro se obtenía agregando cuatro partes de agua hirviendo y cuatro partes de hielo,

2 Para Anaxímenes lo caliente y lo frío son estados comunes de la materia. Consideraba que lo comprimido y condensado era frío, y que lo raro y "laxo" era caliente, por tanto, según él, la rarefacción daba cuenta del proceso mediante el cual se calentaban las cosas, hasta quedar convertidas en vapor. Actualmente este fenómeno puede equipararse con el de la evaporación. la que aplicó principalmente en la medicina (Lynds).

Es de resaltar que durante este periodo ya se apreciaba que algunos de los fenómenos físicos, como la dilatación de sólidos y líquidos, y la expansión térmica del aire y el vapor, dependían del calor, pero no se prestaba atención a mirar dónde se encontraban localizadas las temperaturas porque no era parte de las cualidades referidas en la teoría aristotélica; estas ideas eran aplicadas en la fabricación de máquinas para la diversión, una de ellas es la llamada Aeolipia (figura 1) que, según García (1997) , es construida por Hero de Alejandría (130 a.C) y es la primera máquina térmica de la cual se tiene conocimiento. Esta funciona como una turbina de vapor, que consiste en un globo hueco que se soporta sobre un pivote de madera de tal manera que pueda girar alrededor de dos brazos, uno de los cuales debe ser hueco. En uno de los brazos debe suministrarse vapor de agua, el cual a su vez escapa del globo por dos tubos doblados orientados tangencialmente y en direcciones opuestas, los que están colocados en los extremos del diámetro, perpendicular al eje de rotación del globo. Cuando el vapor es expulsado por los tubos, debido a las fuerzas que allí actúan, este empieza a girar sobre su propio eje.

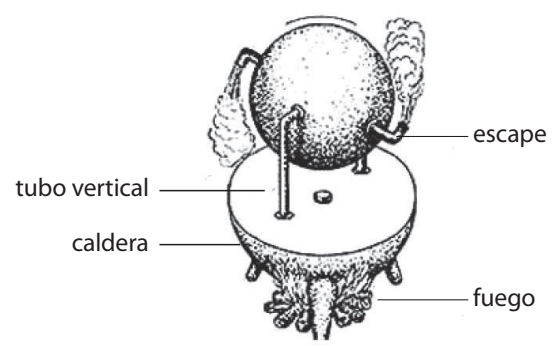

Figura 1 


\section{El alcahesto}

Las ideas de Aristóteles comienzan a ser cuestionadas a mediados del siglo XVI, cuando se propone la existencia de una quinta esencia de la materia -última esencia de la materia- (Taton, 1972), idea que luego de ser estudiada fue transformándose rápidamente en la existencia de un agente universal responsable de todas las reacciones químicas. Más tarde a este agente se le atribuyó la característica de transformar la apariencia física de los cuerpos, por ejemplo, la evaporación del agua, al cambiar de líquido a gaseoso.

Este investigador señala que, en medio de este ambiente, Van Helmont (1577-1644) al realizar observaciones acerca de la calcinación del carbón y el azufre, encuentra una contradicción entre los cuatro elementos con la experiencia, pues el fuego no es un elemento en sí, sino un actor de transformación. A su vez, dedujo que el humo es un gas, al igual que la llama del fuego, la cual nace y desaparece sin tener carácter corporal. Así, al agente universal que produce las reacciones químicas y las transformaciones físicas él lo denominó Alcahesto.

El experimento que utilizó para esto fue la observación del crecimiento de una planta de sauce, crecimiento que atribuyó al agua que él regó durante cinco años, concluyendo así que el agua podía transmutarse en madera y en otros cuerpos pesados como los metales (Taton, 1972).

La teoría del alcahesto -la cual consiste en buscar el agente universal generador de todas las reacciones químicas- fue refutada, bajo la argumentación de que si él podría transformar o disolver cualquier material, entonces debía transformar o disolver el recipiente que lo contenía, siendo imposible conseguirlo, por lo que los argumentos de Van Helmont perdieron fuerza y la mayoría de sus contemporáneos mostraron prudencia y reserva respecto de él.

Así mismo, Taton (1972) menciona que Robert Boyle (1627-1691), como Van Helmont, contemporáneo de Boyle, negó al fuego todo carácter corpóreo y rechazó la concepción de que los otros tres elementos constituyeran la materia. También consideraba que debía existir cierta unidad de la materia, lo que implicaba que ella debería estar compuesta por corpúsculos. De esta forma en el pensamiento de este físico, el concepto de elemento, que él entendió como término último de la descomposición química, no tenía en cuenta el agente universal al cual asociarle una transformación física o química, sino que las variaciones de las partículas y sus movimientos podrían dar cuenta de los diferentes aspectos de los elementos.

Muchos de los escritos de Boyle fueron leídos y discutidos, y aunque las ideas que aportó y criticó de los antiguos sistemas tomaron auge en sus contemporáneos, no se logró reducir la tendencia de los químicos de la época a promover un agente universal reconocido por todos.

Mientras se desarrollaron discusiones en el siglo XVII y los primeros años del XVIII sobre la estructura de la materia, un acontecimiento importante, la teoría del flogisto, introdujo en la doctrina química la tan anhelada unidad.

\section{El flogisto}

Según Efront (1971), la idea de que el agente universal no podía ser sino el principio del fuego fue enunciada por 
Joachim Becher (1635-1682) para ello propuso dos componentes de la materia, agua y tierra, distinguiendo tres tipos de la última: tierra vitrificable, tierra materia y tierra inflamable; Stalh dio a esta última el nombre de flogisto. Este no debe ser confundido con el fuego material, el que se manifiesta en la llama y en el calor cuando se producen combustiones. Él es un elemento inaccesible que poseen todos los cuerpos combustibles.

Al momento de una combustión el flogisto se desprende de los cuerpos, y esa pérdida de flogisto es la que explica el cambio de los cuerpos quemados. Por ejemplo, los aceites al ser quemados dan como resultado agua y aceites privados de flogisto.

Uno de los aspectos más interesantes de esta teoría, según Taton (1972), se refiere a la transformación de los metales en cales y la transformación de los cales en metales, proceso que en la actualidad se denomina reducción. Los metales que contenían más flogisto eran más fácilmente transformables, mientras que los que contenían poco flogisto podían considerarse poco transformables, de modo que el metal era considerado un compuesto de cal y flogisto. Stalh (16601734) observó que cuando se calcinaba el metal, exponiéndolo al aire libre, este perdía su flogisto. La explicación a este fenómeno era que el aire ponía en movimiento las partículas del flogisto y cuando el movimiento era tal que las partículas adquirían velocidad suficiente, se desprendía el flogisto del metal.

La transformación de metales en cal no era únicamente obtenida por exposición al aire libre, también podía obtenerse, si se disolvía tal metal en espíritu de nitro (ácido nítrico) y se calcinaba la sal, quedando únicamente la cal del metal. Cuando esto ocurría, la explicación que se daba era que el metal contenía cierta cantidad de flogisto, y cuando se ponía en contacto con un material como el ácido que no poseía flogisto, la calcinación provocaba la pérdida de flogisto de la mezcla, que se había transformado en cal.

Se observa el proceso inverso; la reducción, solo bastaba suponer que la adición de una cantidad conveniente de flogisto a la cal permitía lograr de nuevo el metal. Por ello, las sustancias ricas en flogisto que les confería su carácter combustible -como el carbón vegetal- eran consideradas especialmente adecuadas para producir estos procesos de obtención del metal.

Esta teoría tuvo inconvenientes, ya que no daba cuenta del porqué al transformarse el metal en cal se producía un aumento de peso en la sustancia, mientras que al transformarse la cal en metal se observaba una disminución de peso. Era toda una contradicción, puesto que el metal estaba compuesto de cal y flogisto.

Stalh, al tratar de explicar este fenómeno concluyó, inicialmente, que la diferencia de peso se debía a que el metal al perder flogisto durante la calcinación quedaba finalmente con la parte más pesada de la sustancia que lo componía. Más tarde atribuyó dicho hecho a que al liberarse la parte inflamable -flogisto-, este dejaba vacíos en la materia, los cuales iban a ser comprimidos por el aire, lo que generaba, a su vez, que la sustancia aumentara su peso.

A pesar de las críticas que se desarrollaron en torno a esta teoría, el problema que se planteaba no era rechazarla por 
el hecho de no explicar claramente el aumento de peso de los metales calcinados, sino cómo hacer entrar el fenómeno en el ámbito de la teoría. Esta actitud era producto de que a la teoría del flogisto no había otra suficientemente estructurada que se le opusiera; este hecho provocó que hasta finales del siglo XVII los químicos acogieran la teoría del flogisto porque respondía a sus expectativas.

Es necesario resaltar aquí, como lo mencionan Rius de Repien y Castro (1995), que en el transcurso del siglo XVII se oponían dos teorías sobre el calor, la del flogisto, y la que razonaban los seguidores de los atomistas griegos, quienes admitían la corporeidad del fuego, considerando que este se constituía por partículas pequeñas, ligeras y sutiles, que tenían a su vez una enorme movilidad para penetrar en la materia en sus diferentes estados, capaces de operar simplemente con su presencia. Las propiedades atribuidas a esta materia del fuego eran, en primer lugar, que se encontraba formada por átomos sutiles que tienen peso $y$, en segundo lugar, materia que se convertía en un fluido indestructible e inmaterial, el calórico.

\section{El calórico}

Lavoisier y sus discípulos fueron los defensores de la teoría del calórico. Propusieron que no había necesidad de un agente hipotético para explicar las reacciones químicas, así el flogisto que había sido el principio del fuego, la luz, y del negro del humo, se transformó en hidrógeno mismo; por tanto, para explicar el fenómeno del aumento del peso de la sustancia cuando se calcinaba, comenzó a elaborar y a defender la idea de que ese aumento se debía a la fijación de una porción del aire atmosférico por parte del metal, de modo que se liberaba la materia del fuego o calórico y se formaba la cal correspondiente (Mallove, 2005).

Entre 1775 y 1777 Lavoisier elaboró una teoría de los gases, en las que introducía el principio del calórico. En este periodo surgía el concepto de temperatura y empezaron a construirse termómetros, para medir la frialdad de las cosas. Joseph Black (1728-1799) utilizó estos termómetros para estudiar el calor, observando cómo las diferentes sustancias que se encontraban a desiguales temperaturas tendían a llegar a un equilibrio cuando se les ponía en contacto.

A partir de la idea de Black se afianzó la teoría que defendía la existencia de un fluido invisible que entraba y salía de una sustancia aumentando o disminuyendo su temperatura. La teoría del calórico se basaba en dos premisas fundamentales: i) El fluido no se crea ni se destruye, ii) La cantidad de calórico transportado hacia o desde el objeto es directamente proporcional a la masa y a la temperatura del objeto. De esta forma, al introducir más calórico en una sustancia, esta se calentaba, hasta que finalmente el calórico se desbordaba y fluía en todas direcciones. Esta era la razón por la cual la calidez de un objeto al rojo vivo se dejaba sentir a gran distancia; el calor del sol, por ejemplo, se notaba a 150 millones de kilómetros. Al poner en contacto un objeto caliente con otro frío, el calórico fluía desde el primero al segundo. Ese flujo hacía que el objeto caliente se enfriara y que el frío se calentara (Taton, 1972).

Mediante la teoría del calórico se explicaban hechos como la dilatación y 
la contracción térmica. Así, calentar un cuerpo era lo mismo que darle calórico y como resultado se evidenciaba la dilatación del mismo; lo contrario ocurría al enfriar el cuerpo, pues el calórico se excluía haciendo que se contrajera. Para dar una razón por la cual una sustancia podía estar en los estados sólido, líquido y gaseoso, esta teoría se basa en la cantidad de calórico. Para una sustancia gaseosa, por ejemplo, se decía que poseía gran cantidad de calórico porque poseía un gran volumen específico, mientras que los sólidos y líquidos contenían menos calórico y por ello ocupaban menos volumen. Cuando un gas se comprimía, el calórico quedaba almacenado en un volumen más pequeño, lo que ocasionaba un crecimiento de la densidad y, por tanto, un aumento de temperatura, al expansionar el gas, la temperatura disminuía.

Esta teoría, aunque explicaba fenómenos como calor, temperatura y combustión, no fue capaz de determinar el peso del calórico al calentar o enfriar los cuerpos.

Como lo menciona Mallove (2005), durante este periodo se observó que las superficies expuestas a rozamientos se calentaban si no estaban suficientemente lubricadas. Los seguidores de la teoría de calórico argumentaron que el calor por fricción podía explicarse como una pérdida del fluido calórico, es decir, el rozamiento obligaba al calórico a salir del material. En 1798 se demostró que ésta explicación no era correcta, mediante la famosa experiencia realizada durante la fabricación de un arsenal de cañones en Baviera; como es lógico, el cañón y el taladro se calentaban y había que estar suministrando constantemente agua fría para refrigerarlos. Rumford vio que el calórico no se desprendía, por la rotura del metal, y que quizá no procedía siquiera de este. El metal estaba inicialmente frío, por lo cual llegó a concluir que no podía contener mucho calórico y, aun así, parecía que el calórico fluía a gran cantidad. Para medir el calórico que salía del cañón, calculó cuánto se calentaba el agua utilizada para refrigerar el taladro y el cañón, y llegó a la conclusión de que si todo ese calórico se reintegraba al metal, el cañón se fundiría.

Rumford finalmente se convenció de que el calor no era un fluido, sino una forma de movimiento. A medida que el taladro rozaba contra el metal, su movimiento se convertía en rápidos y pequeñísimos movimientos de las partículas que constituían el bronce. Igual daba que el taladro cortara o no el metal; el calor provenía de esos pequeñísimos y rápidos movimientos de las partículas, y, como es natural, seguía produciéndose mientras girara el taladro. La producción de calor no tenía nada que ver con ningún calórico que pudiera haber o dejar de haber en el metal.

\section{La energía}

Rius de Repien y Castro (1995) muestran que de la idea de Rumford se concluye la posibilidad de generar por rozamiento una cantidad ilimitada de calor, lo que se explica en la teoría mecanicista, pues la energía mecánica podía llegar a transformarse en calor, hecho que no era fácilmente argumentable con la teoría del calórico. Los paradigmas de la época no permitieron que sus ideas se desarrollaran sino hasta 1820.

La teoría mecánica del calor tomó cuerpo al enunciarse la idea de que había algún tipo de interrelación entre 
el calor y el trabajo. Esta idea culmina con los trabajos de Joule (1818-1889), que establecen que el calor y el trabajo no son más que manifestaciones de la energía. Se postula que si bien la energía mecánica y la energía térmica pueden transformarse una en otra, la energía en sí no podía ser creada ni destruida.

Una segunda hipótesis interesante es que la energía térmica podía solo ser convertida en un porcentaje en trabajo, pero el trabajo puede ser totalmente convertido en calor. Finalmente se comprobó que el calor no podía ser entendido como una sustancia material, sino que es una forma de energía. Es necesario tener en cuenta que el calor no es la energía que contiene un cuerpo, sino la cantidad de energía que pasa de un cuerpo caliente a otro frío o menos caliente. Las medidas del equivalente mecánico del calor señalaron el fin de la teoría del calórico. De todo esto surge la termodinámica y de ella las máquinas térmicas (Rius de Repien y Castro, 1995).

En la misma época en que se inicio la termodinámica estaba desarrollándose la teoría molecular de la materia, que permite formarse una idea coherente del calor y de los fenómenos que intervienen. Algunas de sus premisas son: i) todos los cuerpos están formadas por partículas pequeñísimas, las moléculas, ii) las moléculas no ocupan todo el volumen del cuerpo; entre ellas hay espacios vacíos, iii) entre moléculas se ejercen fuerzas de cohesión, y iv) las moléculas están en movimiento.

Como lo menciona Fuente (2000: 2) la teoría cinética molecular explicará entonces el fenómeno del calor como:

[...] el calor de un cuerpo está direc-

tamente relacionado con la energía

cinética o de movimiento de las moléculas que lo componen; cuanto mayor es la energía cinética más caliente está el cuerpo; cuanto más caliente está una cosa, más rápidamente se mueven sus partículas. Como las moléculas siempre se hayan en movimiento, todo lo existente contiene algún calor. Incluso lo que parece frío encierra cierta medida de temperatura pues sus moléculas se mueven [...].

Esta es precisamente la idea que se tiene actualmente de lo que hace que un cuerpo esté más caliente que otro. La teoría cinética de los gases explicaba muchos de los fenómenos que por medio de la teoría del calórico no podían ser explicados; por ejemplo, para los seguidores de la teoría del calórico, se consideraba la transferencia de calor como un fluido que entraba y salía de los cuerpos para calentarlos o enfriarlos, mientras que para los de la teoría cinética, dicha transferencia se argumenta por el hecho de que las moléculas tienen movimiento. Así, al colocar juntos dos cuerpos con diferentes temperaturas, las moléculas en movimiento del cuerpo más caliente chocan con las del objeto frío, en el cual sus moléculas se desplazan más lentamente, provocando que las moléculas que llevan mayor velocidad la disminuyan, y las del cuerpo frío que viajaban más despacio adquieran una velocidad mayor que la inicial, por esto es que decimos que hay un flujo de calor del cuerpo más caliente al más frío.

\section{Conclusiones}

Se evidencia que el concepto de calor se ha explicado desde dos modelos: i) el sustancialista, que le asigna entidades similares a las de un fluido, y ii) el dinámico, que lo relaciona con el 
movimiento, siendo este último modelo la base fundamental de la concepción actual de calor.

Las concepciones de calor que se explican desde el modelo sustancialista -los cuatro elementos, alcahesto, flogisto y calórico- se caracterizan por considerar que existe una sustancia dentro del mismo cuerpo a la que se le atribuye que éste esté o más caliente o más frío.

La concepción que se explica desde el modelo dinámico - energía - se caracteriza por postular que el calor es: i) el intercambio de energía del sistema con el medio o sus alrededores, y/o ii) las fuerzas o el movimiento de las partículas, involucrando la velocidad de reacción.

La ruptura epistemológica está dada por la naturaleza de estos dos modelos que explican el calor.

El diseño de situaciones para el aula debe involucrar, necesariamente, el uso de los dos modelos mediante los que se explica el concepto de calor, pues la ruptura epistemológica se da en el cambio de una concepción a otra.

\section{Referencias}

Álvarez, M. (2005). Experiencias y perspectivas de la introducción de la historia de las ciencias en la enseñanza secundaria. Enseñanza de las Ciencias, (extra). Disponible en: http://ensciencias.uab.es/webblues/www/congres2005/material/ Simposios/03_Modelos_y_model/ Alvarez_776B.pdf. Fecha de consulta: 21 de octubre de 2006.

Anaconda, M. (2003). Historia de las Matemáticas en la Educación Matemática. Revista EMA, 8 (1), 30-46.

Cubillos, G. (2002). Epistemología e historia en la pedagogía de las ciencias. Revista innovación y ciencia, 10 (3 y 4), 21-25.

Del Río, J. (1997) Historia de las matemáticas. Implicaciones didácticas. Revista Suma, 26, 33-38.
Efront, A. (1971). El mundo del calor. Santander, Buenos Aires: Editorial Bell.

Fuente, A. (2000). El calor. Disponible en: http://html.rincondelvago.com/ calor_13.html. Fecha de consulta 21 de octubre de 2006.

García Martínez, A. (2005). Aportes del estudio histórico de instrumentos científicos a la formación del profesorado de ciencias. Enseñanza de las ciencias, (extra). Disponible en http://ensciencias.uab.es/webblues/www/congres2005/material/ Simposios/03_Modelos_y_model/ Garcia_791.pdf. Fecha de consulta: 12 de octubre de 2006.

García, L. (1997). De la máquina de vapor al cero absoluto (calor y entropía). México: Fondo de Cultura Económica. 
Lynds, B. (2006). About Temperature. Traducción por Riverol C. Disponible en http://eo.ucar.edu/skymath/acerca. html. Fecha de consulta: 15 de octubre de 2006.

Mallove, E. (2005). Los misterios y los mitos del calor: Una breve historia de lo caliente y lo frío. Revista Attos IV. Publicado en su versión original en Infinite Energy 37, mayo-Junio de 2001. Disponible en www.attos.com. Fecha de consulta: 1 de septiembre de 2006.
Quintanilla, M. (2005) Historia de la ciencia y formación del profesorado: una necesidad irreductible. Revista TED (extra), 34-43.

Rius de Repien, M. y Castro, C. (1995). Calor y movimiento. México: Fondo de Cultura Económica.

Taton, R. (1972). Historia general de las ciencias, vol. II. Barcelona: Ediciones Destino. 